\title{
HILBERT'S CHARACTERISTIC FUNCTION AND THE ARITHMETIC GENUS OF AN ALGEBRAIC VARIETY
}

BY

\section{H. T. MUHLY AND O. ZARISKI( $\left.{ }^{1}\right)$}

1. Introduction. The properties of the characteristic function of Hilbert associated with a doubly homogeneous ideal $\mathfrak{a}$ in a ring $R=k[X, Y]$ of polynomials in two sets of indeterminates $(X)$ and $(Y)$ were studied by van der Waerden in $[1]\left({ }^{2}\right)$. Since such an ideal can be regarded as defining an algebraic correspondence between the varieties $U=V(\mathfrak{a} \cap k[X])$ and $V=U(\mathfrak{a} \cap k[Y])$, van der Waerden's results can be looked upon as belonging to the general theory of algebraic correspondences. In case $U$ and $V$ are birationally equivalent normal varieties $\left({ }^{3}\right)$, further results can be given. It is the object of this note to study the properties of the Hilbert characteristic function which is associated with such a pair of normal varieties. A new proof of the invariance of the arithmetic genus of two- and three-dimensional varieties as well as a proof of the Riemann-Roch theorem for a large class of linear systems on an algebraic surface are among the results obtained.

2. Enumerative functions. Let $U$ and $V$ be models of a field $\Sigma$ of degree of transcendency $r$ over a ground field $k$. The field $k$ is assumed to be algebraically closed but otherwise arbitrary. Let $\left(\bar{x}_{1}, \bar{x}_{2}, \cdots, \bar{x}_{g}\right)$ and $\left(\bar{y}_{1}, \bar{y}_{2}, \cdots, \bar{y}_{h}\right)$ be nonhomogeneous coordinates of the generic points of $U$ and $V$ respectively, and let $x_{0}$ and $y_{0}$ be quantities which are algebraically independent over $\Sigma$. Let $x_{i}=x_{0} \bar{x}_{i}, i=1,2, \cdots, g ; y_{j}=y_{0} \bar{y}_{j}, j=1,2, \cdots, h$, and form the ring $\mathrm{D}=k\left[x_{0}, x_{1}, \cdots, x_{g} ; y_{0}, y_{1}, \cdots, y_{h}\right]$. This ring is an integral domain with quotient field $\Sigma\left(x_{0}, y_{0}\right)$. If $R=k\left[X_{0}, X_{1}, \cdots, X_{g}\right.$; $\left.Y_{0}, Y_{1}, \cdots, Y_{h}\right]$ (where $(X)$ and $(Y)$ are indeterminates) and if $\tau$ is the homomorphic mapping of $R$ onto $\mathrm{o}$ which sends $X_{i}$ into $x_{i}, Y_{j}$ into $y_{j}$, then the kernel $a$ of $\tau$ is a doubly homogeneous prime ideal in $R$. The characteristic function $\chi(m, n ; \mathfrak{a})$ defined in [1] as the number of doubly homogeneous forms of degree $m$ in $(X)$ and of degree $n$ in $(Y)$ which are linearly independent (over $k$ ) modulo $\mathfrak{a}$ is an enumerative function determined uniquely by the pair of models $(U, V)$ of the field $\Sigma$. Van der Waerden's main result as-

Presented to the Society, September 10, 1948; received by the editors July 8, 1948.

(1) Research paper done under Office of Naval Research contract N6ori-71, Task Order XVIII at University of Illinois and Harvard University. The ideas expressed in this paper represent the personal views of the authors and are not necessarily those of the Office of Naval Research.

(2) Numbers in brackets refer to the bibliography.

(3) Throughout this paper the term "normal variety" will mean "locally normal variety" in the sense of [5, Definition 3$]$. 
serts that there exist integers $a_{i j}, M, N$ such that

$$
\chi(m, n ; \mathfrak{a})=\sum_{r} a_{i j} C_{m, i} C_{n, j},
$$

when $m \geqq M, n \geqq N$,

where $\sum_{r}$ denotes the sum over all integers $i, j$ such that $i+j \leqq r$. We shall denote the polynomial $\sum_{r} a_{i j} C_{m, i} C_{n, j}$ associated with the pair $(U, V)$ by $\rho(m, n)$.

Let $\left\{A_{m}\right\}\left(^{4}\right)\left(\left\{B_{n}\right\}\right)$ denote the system of $(r-1)$-dimensional subvarieties cut out on $U(V)$ by the hypersurfaces of order $m(n)$ in its ambient space $S_{o}\left(S_{h}\right)$. We regard each of these systems as lying on the join $W$.of $U$ and $V$, and there we consider the minimal sum $\left\{A_{m}+B_{n}\right\}$ of $\left\{A_{m}\right\}$ and $\left\{B_{n}\right\}$ together with the complete sum $\left|A_{m}+B_{n}\right|$. Two enumerative functions $r(m, n)$ and $s(m, n)$ associated with the pair $(U, V)$ are defined as follows:

$$
\begin{aligned}
& r(m, n)=1+\operatorname{dim}\left|A_{m}+B_{n}\right|, \\
& s(m, n)=1+\operatorname{dim}\left\{A_{m}+B_{n}\right\} .
\end{aligned}
$$

Since it is clear from the above definition of the ideal $\mathfrak{a}$ that $\chi(m, n ; \mathfrak{a})$ $=s(m, n)$, equation (2.1) can be written in the form

$$
s(m, n)=\rho(m, n), \quad \text { when } m \geqq M, n \geqq N .
$$

Our main interest is in the function $r(m, n)$. We shall show that under suitable restrictions on the models $U$ and $V$ and on the birational correspondence between them, the minimal sum $\left\{A_{m}+B_{n}\right\}$ is complete for all $m$ when $n$ is large or for all $n$ when $m$ is large. Moreover, for such values of $m$ and $n$, the equations $r(m, n)=s(m, n)=\rho(m, n)$ hold.

3. Varieties of dimension one. As a starting point for induction proofs to be undertaken later we consider the special case in which $\Sigma$ is of degree of transcendency one over $k$. We recall a well known lemma of Castelnuovo which gives a sufficient condition for the minimal sum of two linear series on an algebraic curve to be complete.

Castelnuovo's Lemma. If $g_{\mu}$ is a linear series without fixed points on a curve $\mathrm{I}$, and if $g_{\nu}$ is a complete non-special series on $\Gamma$ which partially contains $g_{\mu}$ and is such that the residual series $g_{\nu}-g_{\mu}$ is non-special, then the minimal sum of $g_{\nu}$ and $g_{\mu}$ is complete.

This lemma leads immediately to the following one.

LEMMA 1. If the variety $V$ (of the pair $(U, V)$ ) is a normal curve, there exists an integer $n_{0}$ such that the minimal sum $\left\{A_{m}+B_{n}\right\}$ is complete if $n \geqq n_{0}$ and $m$ is arbitrary.

Proof. Let $\pi$ be the genus of $\Sigma$ and let $\mu$ and $\nu$ be the orders of $U$ and $V$ respectively. The system $\left\{A_{m}\right\}$ is then a $g_{m \mu}$ while $\left\{B_{n}\right\}$ is a $g_{n \nu}$. Since $V$ is

$\left.{ }^{4}\right)$ The notation $\{C\}$ is used to denote a specified linear system of generic member $C$, and the notation $|C|$ is reserved for the complete system determined by $C$. 
normal, $\left\{B_{n}\right\}$ is complete if $n$ is sufficiently large. We fix $n_{0}$ so that (a) $n \nu-\mu>2 \pi-2$, (b) $g_{n \nu}$ partially contains $g_{\mu}$, (c) $g_{n \nu}$ is complete, when $n \geqq n_{0}$. We proceed by induction on $m$ since for $m=0$ the lemma is trivially true in view of (c). We therefore assume that the minimal sum of $g_{n \nu}$ and $g_{m \mu}$ is a complete series $g_{N}(N=m \mu+n \nu)$. We observe that $g_{N}$ is non-special and that it partially contains $g_{\mu}$. By condition (a), $N-\mu>2 \pi-2$ so that $g_{N}-g_{\mu}$ is also non-special. By Castelnuovo's lemma the minimal sum of $g_{N}$ and $g_{\mu}$ is complete, and since this minimal sum coincides with the minimal sum of $g_{(m+1) \mu}$ and $g_{n v}$, the lemma is proved.

LEMMA 2. Under the hypotheses of Lemma 1, the $\rho$-function associated with the pair $(U, V)$ is given by the formula

$$
\rho(m, n)=m \mu+n \nu-\pi+1 .
$$

Moreover, $r(m, n)=\rho(m, n)$ whenever $m \mu+n \nu>2 \pi-2$.

Proof. If $n \geqq n_{0}$, then $\left\{A_{m}+B_{n}\right\}$ is complete so that $r(m, n)=s(m, n)$ and $r(m, n)=\rho(m, n)$ for large values of $m$ and $n$. Since the complete system $\left|A_{m}+B_{n}\right|$ is non-special when $m$ and $n$ are large, the Riemann-Roch theorem for curves yields $\rho(m, n)=r(m, n)=m \mu+n \nu-\pi+1$. The expression $m \mu+n \nu$ $-\pi+1$ must then give the value of $\rho(m, n)$ for all $m$ and $n$ since $\rho$ is a polynomial. It gives the value of $r(m, n)$ whenever $m \mu+n \nu>2 \pi-2$, q.e.d.

4. The virtual arithmetic genus. A class of varieties which we shall call sectionally normal is defined inductively as follows.

Definition 1. An irreducible 1-dimensional variety is said to be sectionally normal if it is nonsingular. An irreducible $r$-dimensional variety is sectionally normal if almost all $\left({ }^{5}\right)$ of its sections by the hypersurfaces of its ambient space are sectionally normal varieties of dimension $r-1$.

We next define a class of birational transformations.

DEFINITION 2. A birational transformation $T$ defined on an irreducible variety $W \subset S_{n}$ will be called a proper transformation if $T\left(W \cap S_{t}\right)$ is normal for almost all linear subspaces $S_{t}$ of $\left.S_{n}(t=n-r+1), \cdots, n\right)$. In particular, $T(W)$ is required to be normal.

We point out that any normal curve or surface is sectionally normal as is any nonsingular variety of arbitrary dimension $\left(^{6}\right)$; moreover, if $T$ is a regular birational transformation defined on a sectionally normal variety $W$, then $T$ is proper. We shall have occasion to point out in the next section that there are proper transformations which are not regular.

(5) The term "almost all" here means all with the possible exception of a proper algebraic subsystem.

${ }^{(6)}$ It has recently been proved by A. Seidenberg (but not yet published) that almost all hyperplane sections of a normal variety $V$ are normal varieties. This implies that every normal variety is sectionally normal. 
THEOREM 1. If the transformation $T: U \rightarrow V$ is proper then there exists an integer $n_{0}$ such that the minimal sum of $\left\{A_{m}\right\}$ and $\left\{B_{n}\right\}$ is complete when $n \geqq n_{0}, m \geqq 0$.

Proof. The proof is by induction with respect to the dimension $r$ of the field $\Sigma$ of rational functions on $U$ and $V$. By Lemma 1 the theorem is true if $\Sigma$ is of dimension one over $k$ and we assume that it is true if $\Sigma$ is of dimension $r-1$ over $k$. The passage from $r-1$ to $r$ is effected by induction with respect to $m$. Since $T$ is proper, the variety $V$ is normal so that the minimal sum $\left\{A_{m}+B_{n}\right\}$ is complete if $m=0$ and $n$ is sufficiently large. We assume that the minimal sum $\left\{A_{m-1}+B_{n}\right\}$ is complete if $n$ is large.

Let $A$ be an irreducible hyperplane section of $U$ such that $A^{\prime}=T(A)$ is normal, and the transformation $T^{\prime}: A \rightarrow A^{\prime}$ induced by $T$ is proper. (Such hyperplane sections exist in view of the theorem of Bertini [4] and the fact that $T$ is proper( $\left.{ }^{7}\right)$.) If $\left\{\bar{A}_{m}\right\}$ and $\left\{\bar{B}_{n}\right\}$ are the linear systems cut out on $A$ and $A^{\prime}$ by the hypersurfaces of their respective ambient spaces, then these systems are cut out on $A$ and $A^{\prime}$ by the systems $\left\{A_{m}\right\}$ and $\left\{B_{n}\right\}$. By the induction assumption the minimal sum $\left\{\bar{A}_{m}+\bar{B}_{n}\right\}$ is complete if $n \geqq \bar{n}_{0}$. We denote its dimension (increased by one) by $\bar{r}(m, n)$, and we fix $n_{0}\left(\geqq \bar{n}_{0}\right)$ so that $\left\{B_{n}\right\}$ is complete when $n \geqq n_{0}$. If $G$ is the minimal sum of $\left\{A_{m}\right\}$ and $\left\{B_{n}\right\}$, then $G$ cuts out the complete system $\left|\bar{A}_{m}+\bar{B}_{n}\right|$ on $A$, the dimension of which is $\bar{r}(m, n)-1$. The residual system of $G$ with respect to $A$ is the minimal sum $\left\{A_{m-1}+B_{n}\right\}$, which by the induction assumption is complete. Since the dimension of $\left|A_{m-1}+B_{n}\right|$ is given by $r(m-1, n)-1$ we conclude that

$$
1+\operatorname{dim} G=r(m-1, n)+\bar{r}(m, n) .
$$

(7) The theorem of Bertini is proved in [4] under the hypothesis that $k$ is of characteristic zero. In the special case of the hyperplane sections of an irreducible variety the following considerations remove this restriction on the characteristic. Let $V / k$ be an irreducible variety in $S_{n}$ ( $k$ is algebraically closed) and let $p$ be the prime homogeneous ideal of $V$ in $k[Y]\left(=k\left[Y_{0}, Y_{1}, \cdots, Y_{n}\right]\right)$. Let $(u)=\left(u_{0}, u_{1}, \cdots, u_{n}\right)$ be a set of $n+1$ indeterminates (algebraically independent quantities over $k$ ) and let $K$ denote the field generated over $k$ by the quotients of the $u$ 's. If $L$ denotes the linear form $\sum u_{i} Y_{i}$, then it is easily seen that the ideal $(p, L)$ in $k(u)[Y]$ is prime. This implies that the intersection $W$ of $V$ with the hyperplane $L=0$ is irreducible over $K$. The variety $W$ is of dimension $r-1$. If $m$ is the order of $V$, any linear $S_{n-r}$ which is general over $k$ meets $V$ in $m$ distinct points. Hence any linear $S_{n-r+1}$ which is general over $K$ meets $W$ in $m$ distinct points. It follows that also $W$ is of order $m$.

Let $P$ be a general point of $W / K$. Then it is clear that $P$ is also a general point of $V / k$ and that $K$ is a pure transcendental extension of $k(P)$ (of transcendence degree $n-1$ ). Hence $K$ is maximally algebraic in $K(P)$ and therefore $W$ is an absolutely irreducible variety. Also the associated form $F$ of $W$ (in the sense of Chow-van der Waerden) is therefore absolutely irreducible (the coefficients of $F$ may be assumed to be forms in $k[u]$ ). Hence $F$ remains irreducible for almost all specializations $(u) \rightarrow(\bar{u}), u_{i} \in k$. Since in any such specialization, $F$ is specialized to the associated form $\bar{F}$ of the corresponding hyperplane section $\bar{W}$ of $V$ (here $\bar{W}$ is to be thought of as a cycle, not a variety), it follows at once that almost all hyperplane sections of $V$ are irreducible varieties of order $m$. 
However, if $H$ is the complete system $\left|A_{m}+B_{n}\right|$ determined by $G$, the same reasoning applied to $H$ would lead to the conclusion that

$$
1+\operatorname{dim} H=r(m-1, n)+\bar{r}(m, n) .
$$

It follows that $G=H$, q.e.d.

COROLlaRY 1. Under the hypothesis of Theorem 1, the function $r(m, n)$ satisfies the addition formula

$$
r(m, n)=r(m-1, n)+\bar{r}(m, n)
$$

when $n \geqq n_{0}$.

COROLlARY 2. The $r$ - and $\rho$-functions associated with the pair $(U, V)$ are equal when $n \geqq n_{0}$ and $m$ is arbitrary.

Proof. Let $\bar{\rho}(m, n)$ be the $\rho$-function associated with the pair $\left(A, A^{\prime}\right)$ introduced above. Since the statement is true for varieties of dimension one by Lemma 2, we assume for purposes of induction that $\bar{r}(m, n)=\bar{\rho}(m, n)$ if $n \geqq \bar{n}_{0}$ and $m \geqq 0$. Since the minimal sum $\left\{A_{m}+B_{n}\right\}$ is complete, $r(m, n)$ $=s(m, n)$, so that if both $m$ and $n$ are large, $r(m, n)=\rho(m, n)$. It follows from (4.1) that

$$
\rho(m, n)=\rho(m-1, n)+\bar{\rho}(m, n)
$$

if both $m$ and $n$ are large, and since the $\rho$-functions are polynomials, (4.2) is valid for all values of $m$ and $n$. Equations (4.1) and (4.2) together imply that if $r(m, n)=\rho(m, n)$ then also $r(m-1, n)=\rho(m-1, n)$. This completes the proof.

Let $W$ be a normal variety defined by a homogeneous prime ideal $p$ in the ring $S=k\left[X_{0}, X_{1}, \cdots, X_{h}\right]$, and let $\left\{C_{n}\right\}$ be the linear system cut out on $W$ by the hypersurfaces of order $n$ in the ambient space $S_{h}$ of $W$. If $r(n)-1$ is the dimension of the complete system $\left|C_{n}\right|$ and if $\chi(n ; \mathfrak{p})$ is the number of forms of degree $n$ in $S$ which are linearly independent modulo $\mathfrak{p}$, then $r(n)$ $=\chi(n ; \mathfrak{p})$ if $n$ is sufficiently large, since $W$ is normal. The function $\chi(n ; \mathfrak{p})$ is a polynomial in $n$ if $n$ is large;

$$
\chi(n ; \mathfrak{p})=\rho(n)=\sum_{i=0}^{r} a_{i} C_{n, i}
$$

where the coefficients, $a_{0}, a_{1}, \cdots, a_{r}$ are integers uniquely determined by $W$, and $r$ is the dimension of $W$. We call $\rho(n)-1$ the virtual dimension of $\left|C_{n}\right|$, and note that for large values of $n$ the effective dimension $r(n)-1$ equals the virtual dimension $\rho(n)-1$.

DEFINITION 3. The virtual arithmetic genus $p_{a}(W)$ of the normal r-dimensional variety $W$ is the integer $(-1)^{r}\left(a_{0}-1\right)$.

TheOREM 2. If $U$ and $V$ are normal models of $\Sigma$ and if $T: U \rightarrow V$ and 
$T^{-1}: V \rightarrow U$ are both proper transformations, then $p_{a}(U)=p_{a}(V)$.

Proof. Let $r_{1}(m), \rho_{1}(m) ; r_{2}(n), \rho_{2}(n)$ be the effective and virtual dimensions (increased by one) of $\left|A_{m}\right|$ and $\left|B_{n}\right|$ respectively, and let $r(m, n), \rho(m, n)$ be the $r$ - and $\rho$-functions associated with the pair $(U, V)$. Let $\rho_{1}(m)$ $=\sum c_{1 i} C_{m, i}, \rho_{2}(n)=\sum c_{2 j} C_{n, j}$. By definition, $r_{1}(m)=r(m, 0), r_{2}(n)=r(0, n)$. Since both $T$ and $T^{-1}$ are proper, it follows by Corollary 2 of Theorem 1 that there exist integers $m_{0}$ and $n_{0}$ such that

$$
\begin{array}{rlrl}
r(m, 0) & =\rho(m, 0), & & \text { if } m \geqq m_{0}, \\
r(0, n)=\rho(0, n), & & \text { if } n \geqq n_{0} .
\end{array}
$$

It follows that

$$
\rho_{1}(m)=\rho(m, 0), \quad \rho_{2}(n)=\rho(0, n),
$$

for all values of $m$ and $n$. If $a_{00}$ is the constant term in $\rho(m, n)$, then $c_{10}=a_{00}$ $=c_{20}$. It follows that $p_{a}(U)=p_{a}(V)$, q.e.d.

COROLLARY 1. If $U$ and $V$ are sectionally normal models in regular birational correspondence, then $p_{a}(U)=p_{a}(V)$. In other words, the virtual arithmetic genus of a sectionally normal model is a relative birational invariant.

Proof. We have pointed out above that a regular birational transformation defined on a sectionally normal model is proper. Since the inverse of a regular transformation is also regular, the corollary follows.

5. Varieties of dimension two and three. In this article we confine our attention to the cases in which $\Sigma$ is of dimension two or three over $k$ and we restrict our remarks to nonsingular models of such fields. We assume throughout the remainder of the text that $k$ is of characteristic zero.

If $U$ and $V$ are nonsingular models of $\Sigma$ and if $V$ dominates $U, U<V\left({ }^{8}\right)$, then it is immediately clear that $T: U \rightarrow V$ is proper. In fact, the system $T\left(\left\{A_{1}\right\}\right)$ on $V$ has no base points (since $U<V$ ) and since $V$ has no singularities, the general member of $T\left(\left\{A_{1}\right\}\right)$ has no singularities in view of Bertini's theorem [6]. If $\Sigma$ is of dimension two this remark suffices to prove that $T$ is proper. If $\Sigma$ is of dimension three we observe in addition that a generic $A_{1}$ and its transform $T\left(A_{1}\right)$ are nonsingular and that $A_{1}<T\left(A_{1}\right)$. Hence $T$ induces a proper transformation on a generic $A_{1}$ so that it is itself proper.

We consider quadratic and monoidal transformations in the next two lemmas. These terms are used in the sense of [5, article 11].

LEMMA 3. If $U$ and $V$ are nonsingular, and if $V$ is obtained from $U$ by a

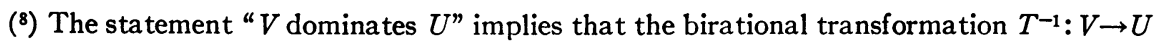
has no fundamental points on $V$; or equivalently, the local ring $Q\left(P^{\prime}\right)$ contains the local ring $Q(P)$, when $P(\subset U)$ and $P^{\prime}(\subset V)$ are a pair of corresponding points in the birational correspondence $T$. This relationship is denoted by the symbols $U<V$. 
quadratic transformation with center at a point $P$ of $U$, then both $T: U \rightarrow V$ and $T^{-1}: V \rightarrow U$ are proper transformations.

Proof. In this case $U<V$ so that $T$ is proper. Let $\left(\eta_{0}, \eta_{1}, \cdots, \eta_{0}\right)$ be homogeneous coordinates of the general point of $U$ and let $\mathfrak{p}$ be the prime ideal of $P$ in the ring $0=k[\eta]$. If $\phi_{0}, \phi_{1}, \cdots, \phi_{h}$ form a linear basis for the forms of degree $\nu$ in $\mathfrak{p}$, and if $\nu$ is sufficiently large, then the ideal $\mathfrak{a}=\sum \mathfrak{D} \phi_{i}$ differs from $\mathfrak{p}$ by at most an irrelevant component and $\left(\phi_{0}, \phi_{1}, \cdots, \phi_{h}\right)$ can be regarded as the general point of $V$. Hence the linear system defined by $\phi(\lambda)=0$, where $\phi(\lambda)=\lambda_{0} \phi_{0}+\lambda_{1} \phi_{1}+\cdots+\lambda_{h} \phi_{h}, \lambda_{i} \in k$, is the inverse transform of the system $\left\{B_{1}\right\}$ of hyperplane sections of $V$. The point $P$ is the only base point of this system, and since $U$ is nonsingular, the generic member of this system has no singular points except possibly at $P$ itself. If we assume that $P$ is not on the hyperplane section $\eta_{0}=0$, and if we put $\psi_{i}=\phi_{i} / \eta_{0}^{\nu}$, then the quantities $\psi_{0}, \psi_{1}, \cdots, \psi_{h}$ will form a basis for the prime ideal of $P$ in the quotient ring $Q(P / U)$. It follows that among the $\psi_{i}$ there is at least one whose leading form at $P$ is linear so that almost all members of $\{\phi(\lambda)\}$ have a simple point at $P$ (see [6, p. 137]). The generic member of $\{\phi(\lambda)\}$ is therefore nonsingular and hence normal. This proves that $T^{-1}$ is proper if $U$ and $V$ are surfaces.

If $\Sigma$ is of dimension three, it is necessary to prove in addition that the general characteristic curve of the system $\{\phi(\lambda)\}$ is nonsingular, since such a curve is the transform by $T^{-1}$ of a section $V \cap S_{h-2}$, where $S_{h}$ is the ambient space of $V$. However, it is proved in [5] that a quadratic transformation with center at a point $P$ of $U$ induces a quadratic transformation on any subvariety of $U$ through $P$. If we apply this remark to a generic member $\phi$ of the system $\{\phi(\lambda)\}$ we conclude as above that the generic characteristic curve $\bar{\phi} \cap \phi(\lambda)$ has no singularities, q.e.d.

If $U$ and $V$ are three-dimensional varieties, and if $T$ is a monoidal transformation with center along an irreducible curve $\Delta \subset U$, then since $U$ is nonsingular, every point of $\Delta$ is a simple point of $U$. If in addition every point of $\Delta$ is a simple point of $\Delta$, then we shall call $T$ a nonsingular monoidal transformation.

LEMMA 4. If $U$ and $V$ are nonsingular and if $T: U \rightarrow V$ is a nonsingular monoidal transformation, then $T$ and $T^{-1}$ are proper.

Proof. Since $U<V, T$ is proper. The proof that $T^{-1}$ is proper is similar to the proof given in Lemma 3 for quadratic transformations. We use the same notations as in Lemma 3, except that now $\mathfrak{p}$ is the prime ideal of $\Delta$ in $\mathfrak{D}$. The general member of the system $\{\phi(\lambda)\}$ has no singularities except possibly at points of $\Delta$. If $P$ is a point of $\Delta$ not on the surface $\eta_{0}=0$, then $\psi_{0}, \psi_{1}, \cdots$, $\psi_{h}$ will form a basis for the prime ideal of $\Delta$ in $\mathfrak{T}=Q(P / U)$. Then, since $P$ is simple both for $\Delta$ and for $U$, it follows that among the $\psi_{i}$ there will be at least 
one whose leading form at $P$ is linear (see [5, footnote 34$]$ ). Hence $P$ cannot be a singular base point of $\{\phi(\lambda)\}$. It follows that the generic member of $\{\phi(\lambda)\}$ is nonsingular.

To complete the proof we must show that the generic characteristic curve of $\{\phi(\lambda)\}$ is nonsingular, and for this it suffices to show, in view of Bertini's theorem, that the system cut by $\{\phi(\lambda)\}$ on a generic member $\phi$ (outside the fixed curve $\Delta$ ) has no base points on $\Delta$. The total transform $T(\Delta)$ of $\Delta$ is an irreducible surface $F$ on $V$ which is "ruled" by a pencil $\{f\}$ of rational curves. The curves $f$ are in $1: 1$ correspondence with the points of $\Delta$ and each $f$ is the total transform of the point of $\Delta$ to which it corresponds [7, article 5]. We choose a hyperplane section $B_{1}$ of $V$ such that the section $F \cap B_{1}$ is an irreducible curve $\Gamma$ which is not a component of any member of the pencil $\{f\}$. We further assume that $T^{-1}\left(B_{1}\right)$ is a nonsingular irreducible member $\bar{\phi}$ of $\{\phi(\lambda)\}$. If $\phi(\lambda) \in\{\phi(\lambda)\}$, we write $\phi(\lambda) \cap \bar{\phi}=\Delta+R(\lambda)$. Let us assume that $P \in \Delta$ is a base point of the residual system $\{R(\lambda)\}$. It then follows that every hyperplane section $B_{1}^{\prime}$ of $V$ meets the section $B_{1}$ in a point of the variety $T(P) \cap \Gamma$. Since $\Gamma$ is not a component of any member of $\{f\}$, it follows that $T(P) \cap \Gamma$ consists of a finite set of points, so that the conclusion that every hyperplane $B_{1}^{\prime}$ meets $\Gamma$ in a point of $T(P) \cap \Gamma$ is absurd, q.e.d.

These lemmas yield the following further corollary to Theorem 2 .

COROLlARY 2. The virtual arithmetic genus of a nonsingular model of a field $\Sigma$ of dimension two or three over $k$ is invariant under quadratic and nonsingular monoidal transformations.

The considerations of part IV of [7] show in particular that if $U$ and $V$ are nonsingular models of a three-dimensional field $\Sigma$, then there exist models $U_{1}$ and $V_{1}$ in regular birational correspondence such that $U_{1}\left(V_{1}\right)$ is obtained from $U(V)$ by a sequence of quadratic and nonsingular monoidal transformations. It follows that $p_{a}(U)=p_{a}\left(U_{1}\right)=p_{a}\left(V_{1}\right)=p_{a}(V)$. Since a similar statement is true for nonsingular surfaces (see [3]) we can assert the following theorem.

THEOREM 3. If $\Sigma$ is a field of dimension two or three over $k$, then any two nonsingular models of $\Sigma$ have the same virtual arithmetic genus.

The virtual arithmetic genus of a nonsingular model can therefore be regarded as a character of the field rather than of a particular model. This character is called the arithmetic genus of $\Sigma$ and is denoted by $p_{a}(\Sigma)$.

6. Normal surfaces. Our objective in this section is to show that if $U$ is any normal model of a field $\Sigma$ of dimension two over $k$, then the virtual arithmetic genus of $U$ is not less than the arithmetic genus of $\Sigma, p_{a}(U)$ $\geqq p_{a}(\Sigma)$. Since it is known that there exists a nonsingular model $V$ of $\Sigma$ such that $V>U[2]$, the following theorem will yield the desired result. 
THEOREM 4. If $U$ and $V$ are normal models of $\Sigma$ ( $\Sigma$ of dimension two), and if $U<V$, then $p_{a}(U) \geqq p_{a}(V)$.

The major portion of the proof of this theorem is contained in the following two lemmas.

Lemma 5. Let $\Gamma$ be an irreducible algebraic curve of order $\nu$ in $S_{n}$, and let $g_{m \nu}$ be the series cut out on $\Gamma$ by the hypersurfaces of order $m$ in $S_{n}$. If $g_{\mu}$ is a linear series on $\Gamma$ without fixed points, then there exists an integer $M$ such that if $m \geqq M$, the deficiency of the minimal sum of $g_{m \nu}$ and $g_{\mu}$ is not greater than the deficiency of $g_{m v}$, in symbols, $\delta\left(g_{m \nu}+g_{\mu}\right) \leqq \delta\left(g_{m v}\right)$.

Proof. Let $G_{\mu}$ be a set of the series $g_{\mu}$ and choose nonhomogeneous coordinates on $\Gamma$ so that no point of $G_{\mu}$ is at infinity. If $\mathfrak{a}$ is the ideal in the ring $\mathfrak{D}=k\left[x_{1}, x_{2}, \cdots, x_{n}\right]$ of nonhomogeneous coordinates on $\Gamma$ which is determined by $G_{\mu}$, then the length of $\mathfrak{a}$ is $\mu$, and there exist $\mu$ elements $\phi_{1}, \phi_{2}, \cdots, \phi_{\mu}$ in $\mathfrak{D}$ which form an independent $k$-basis for $\mathfrak{D}$ modulo $\mathfrak{a}$. If $m$ exceeds the degree of each of the polynomials $\phi_{i}$, then the set $G_{\mu}$ will impose $\mu$ independent conditions on the series $g_{m \nu}$. That is, if $\bar{g}_{m \nu}$ is the subsystem of $g_{m \nu}$ consisting of those sets of $g_{m \nu}$ which contain $G_{\mu}$, then $\operatorname{dim} \bar{g}_{m \nu}=\operatorname{dim} g_{m \nu}-\mu$.

Fix $M$ so large that the above condition holds when $m \geqq M$. Increase $M$ if necessary so that $g_{m \nu} \supset g_{\mu}$, and the residual series $g_{m \nu}-g_{\mu}$ is non-special when $m \geqq M$. Let $G_{\mu}^{\prime}$ be another fixed set of $g_{\mu}$ which has no point in common with $G_{\mu}$. Consider the two series $g_{m \nu}+G_{\mu}$ and $g_{m \nu}+G_{\mu}^{\prime}$ obtained by adding the fixed sets $G_{\mu}$ and $G_{\mu}^{\prime}$ to the series $g_{m \nu}$. The union of these two series is contained in the minimal sum of $g_{m \nu}$ and $g_{\mu}$. The common part of these series is the series $\bar{g}_{m \nu}$. Hence

$$
m \nu+\mu-\pi-\delta\left(g_{m \nu}+g_{\mu}\right) \geqq 2\left(m \nu-\pi-\delta\left(g_{m \nu}\right)\right)-\left(m \nu-\pi-\delta\left(g_{m \nu}\right)-\mu\right),
$$

and $\delta\left(g_{m \nu}+g_{\mu}\right) \leqq \delta\left(g_{m \nu}\right)$, q.e.d.

LEMMA 6. If $U$ and $V$ are normal models of $\Sigma$ such that $U<V$, then there exist integers $m_{0}, d$ such that

$$
\rho(m, n)+d \geqq r(m, n) \geqq \rho(m, n)
$$

when $m \geqq m_{0}$ and $n \geqq 0$.

Proof. Since $U<V$, and since $U$ and $V$ are normal, it follows that $T: U \rightarrow V$ is proper, so that by Corollary 2 of Theorem 1 there exists an integer $n_{0}$ such that $r(m, n)=\rho(m, n)$ when $n \geqq n_{0}, m \geqq 0$. Let $B_{1}$ be an irreducible nonsingular hyperplane section of $V$, and let $\bar{B}_{1}=T^{-1}\left(B_{1}\right)$. If $r^{*}(m, n)$ and $\rho^{*}(m, n)$ are the $r$ - and $\rho$-functions associated with the pair $\left(\bar{B}_{1}, B_{1}\right)$, then, by Lemma $2, r^{*}(m, n)=\rho^{*}(m, n)$ for all $m$ if $n$ is large or for all $n$ if $m$ is large. The image of $B_{1}$ on the join of $U$ and $V$ is a normal curve, so that it follows as in the proof of Theorem 1 that $\left|A_{m}+B_{n}\right|$ will cut a complete series on $B_{1}$ if $n$ is large. Since the residual system of $\left|A_{m}+B_{n}\right|$ with respect to $B_{1}$ is 
the complete system $\left|A_{m}+B_{n-1}\right|$, we conclude that $r(m, n)=r(m, n-1)$ $+r^{*}(m, n)$ for large values of $n$. It follows that $\rho(m, n)-\rho(m, n-1)$ $=\rho^{*}(m, n)$ for all values of $m, n$ since the $\rho$-functions are polynomials.

Let $g_{m \nu}$ be the series cut out on $\bar{B}_{1}$ by the system $\left\{A_{m}\right\}$. If we apply Lemma 5 to the case in which the $g_{\mu}$ of that lemma is the series $g_{\nu}$, we find $\delta\left(g_{(m+1) v}\right) \leqq \delta\left(g_{m v}\right)$ for large values of $m$. It follows that $\delta\left(g_{m v}\right)$ remains constant when $m$ is large, say $\delta\left(g_{m v}\right)=\delta$ if $m \geqq M_{0}$. Let $g_{s \mu}$ be the series cut on $B_{1}$ by $\left\{B_{s}\right\}, s=1,2, \cdots, n_{0}$, consider it as a series on $\bar{B}_{1}$, and apply Lemma 5 to each of the series $g_{s \mu}$ in turn. This yields a set of integers $M_{1}, M_{2}, \cdots, M_{n_{0}}$. Let $m_{0}=\max \left(M_{0}, M_{1}, \cdots, M_{n_{0}}\right)$. We can then assert that if $m \geqq m_{0}$, then $\delta\left(g_{m \nu}+g_{s \mu}\right) \leqq \delta, s=1,2, \cdots, n_{0}$.

The system cut by $\left|A_{m}+B_{s}\right|$ on $B_{1}\left(m \geqq m_{0}, s=1,2, \cdots, n_{0}\right)$ contains the minimal sum $g_{m \nu}+g_{s \mu}$ and is totally contained in the complete series determined by $g_{m \nu}+g_{s \mu}$. Since the residual system of $\left|A_{m}+B_{s}\right|$ with respect to $B_{1}$ is the complete system $\left|A_{m}+B_{s-1}\right|$, the following inequalities are valid:

$$
\begin{aligned}
r(m, s-1)+r^{*}(m, s) \geqq r(m, s) \geqq r(m, s-1)+r^{*}(m, s)-\delta, & \\
& s=1,2, \cdots, n_{0} .
\end{aligned}
$$

On combining these inequalities with the equalities $r(m, n)=\rho(m, n), n \geqq n_{0}$, $m \geqq 0 ; r^{*}(m, n)=\rho^{*}(m, n), m \geqq m_{0}, n \geqq 0$, and using the addition formula $\rho(m, n)-\rho(m, n-1)=\rho^{*}(m, n)$, we find

$$
\rho(m, s)+\left(n_{0}-s\right) \delta \geqq r(m, s) \geqq \rho(m, s), \quad s=0,1, \cdots, n_{0} ; m \geqq m_{0} .
$$

If we put $d=\delta n_{0}$, the lemma follows, q.e.d.

Theorem 4 now follows quite readily. As in the proof of Theorem 2, let $r_{1}(m), \rho_{1}(m) ; r_{2}(n), \rho_{2}(n)$ be the effective and virtual dimensions (increased by one) of $\left|A_{m}\right|$ and $\left|B_{n}\right|$, and let

$$
\rho_{1}(m)=\sum_{i=0}^{2} c_{1 i} C_{m, i}, \quad \rho_{2}(n)=\sum_{j=0}^{2} c_{2 j} C_{n, j}, \quad \rho(m, n)=\sum_{2} a_{i j} C_{m, i} C_{n, j} .
$$

Since $r_{2}(n)=r(0, n)=\rho(0, n)$ if $n \geqq n_{0}$, it follows that $c_{20}=a_{00}$. On the other hand, $\rho_{1}(m)=r_{1}(m)=r(m, 0)$ for large values of $m$ so that, by Lemma 6 , $\rho(m, 0)+d \geqq \rho_{1}(m) \geqq \rho(m, 0)$. It follows that the polynomials $\rho(m, 0)$ and $\rho_{1}(m)$ differ only by a constant and that $c_{10} \geqq a_{00}$. Hence $p_{a}(U) \geqq p_{a}(V)$, q.e.d.

If $W$ is an arbitrary normal model of a two-dimensional field $\Sigma$, if the system $\{C\}$ of hyperplane sections of $W$ is of degree $\nu$ and genus $\pi$, and if $p_{a}(W)=p_{a}(\Sigma)+s$, where $s \geqq 0$, then by a straightforward computation it is found that the function $\rho(n)$ associated with $W$ is given by the expression

$$
\rho(n)={ }_{\nu} C_{n, 2}+(\nu-\pi+1) n+p_{a}(\Sigma)+s+1 .
$$

It follows that

$$
\operatorname{dim}|n C|=\nu C_{n, 2}+(\nu-\pi+1) n+p_{a}(\Sigma)+s
$$


if $n$ is sufficiently large. This is the Riemann-Roch theorem for the complete system $|n C|$.

\section{BIBLIOGRAPHY}

1. B. L. van der Waerden, On Hilbert's function, series of composition of ideals, and a generalization of a theorem of Bezout, Proceedings of the Koninklijke Akademie van Wetenschappen, Amsterdam vol. 31 (1928).

2. $\mathrm{O}$. Zariski, The reduction of the singularities of an algebraic surface, Ann. of Math. vol. 40 (1939).

3. - A simplified proof of the resolution of singularities of an algebraic surface, Ann. of Math. vol. 43 (1942).

4. - Pencils on an algebraic variety and a new proof of a theorem of Bertini, Trans. Amer. Math. Soc. vol. 50 (1941).

5. - Foundations of a general theory of birational correspondences, Trans. Amer. Math. Soc. vol. 53 (1943).

6. - The theorem of Bertini on the variable singular points of a linear system of varieties, Trans. Amer. Math. Soc. vol. 56 (1944).

7. - Reduction of the singularities of algebraic three dimensional varieties, Ann. of Math, vol. 45 (1944).

UNIVERSITY OF ILLINOIS,

URBANa, ILL.

HARVARD UNIVERSITY,

Cambridge, Mass. 